Graph 1. Patients with attributable NPSLE were younger, had earlier disease onset, presented higher disease activity, lower damage accrual without taking NP damage into account and more often had increased anti-dsDNA serum concentration.

Table 1. Demographic and laboratory characteristics with disease activity and damage of the study groups, $N(\%)$ or mean( $\pm S D)$.

\begin{tabular}{lcc}
\hline Characteristic & $\begin{array}{c}\text { Patients with } \\
\text { attributed NPSLE } \\
\text { manifestations }\end{array}$ & $\begin{array}{c}\text { Patients without } \\
\text { attributed NPSLE } \\
\text { manifestations }\end{array}$ \\
\hline Patients & $34(23.8 \%)$ & $109(76.2 \%)$ \\
Sex, female & $30(88.2 \%)$ & $102(93.6 \%)$ \\
Age (years) & $37.6( \pm 11.7)$ & $44.3( \pm 13.9)^{\star}$ \\
Age of disease onset (years) & $32.5( \pm 11.4)$ & $37.6( \pm 12.6)^{\star}$ \\
Disease duration (years) & $5.1( \pm 4.1)$ & $6.8( \pm 5.6)$ \\
SLEDAl-2K & $29.2( \pm 10.7)$ & $12.2( \pm 8.1)^{\star}$ \\
patients with clinically active disease (defined as & $34(100 \%)$ & $93(85.3 \%)^{\star}$ \\
SLEDAl-2K $\geq 6$ in clinical manifestations) & & \\
SLEDAl-2K without NP manifestations & $14.8( \pm 8.4)$ & $11.0( \pm 6.7)^{\star}$ \\
PGA & $2.1( \pm 1.0)$ & $1.2( \pm 1.0)^{\star}$ \\
SDI & $0.5( \pm 0.8)$ & $0.7( \pm 1.1)$ \\
SDI without NP damage & $0.3( \pm 0.6)$ & $0.7( \pm 1.1)^{\star}$ \\
low C3/C4 complement component concentration in & $21(61.8 \%)$ & $55(50.4 \%)$ \\
serum & & \\
elevated anti-dsDNA autoantibody concentration in & $27(79.4 \%)$ & $55(50.4 \%)^{\star}$
\end{tabular}

serum

NPSLE - neuropsychiatric systemic lupus erythematosus, SLEDAI-2K - Systemic Lupus Erythematosus Disease Activity Index version 2000, PGA - physician global assessment, SDI - SLICC/ACR (Systemic Lupus International Collaborating Clinics/American College of Rheumatology) Damage Index

${ }^{*} \mathrm{p}<0,05$, Mann-Whitney $\mathrm{U}, \mathrm{x}^{2}$ or Fisher's exact test, as appropriate

Conclusion: Primary NP manifestations in patients with SLE occur mainly in young patients with high disease activity. Cerebrovascular disease, seizures, psychosis and cranial neuropathy are most frequent primary NPSLE manifestations. References:

[1] The American College of Rheumatology nomenclature and case definitions for neuropsychiatric lupus syndromes. Arthritis Rheum. 1999;42(4):599-608.

[2] Bortoluzzi A, Scirè CA, Bombardieri S, Caniatti L, Conti F, De Vita S, et al. Development and validation of a new algorithm for attribution of neuropsychiatric events in systemic lupus erythematosus. Rheumatol Oxf Engl. 2015;54(5):891-8.

Graph 1. Attribution of neuropsychiatric manifestations to systemic lupus erythematosus (\% of attributed manifestations are presented above the bars).

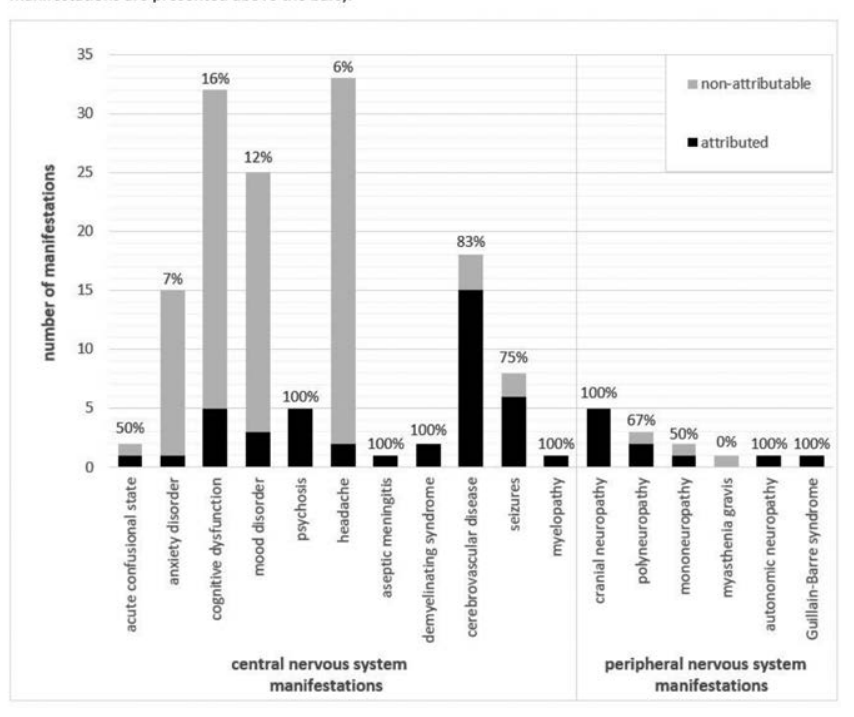

Disclosure of Interests: None declared

DOI: 10.1136/annrheumdis-2020-eular.2470

\section{FRI0185 DIAGNOSTIC SIGNIFICANCES OF BOTH MICRORNA- 146A AND KALLIKREIN-1 IN PATIENTS WITH LUPUS NEPHRITIS}

S. Soliman ${ }^{1}$, A. Higazi ${ }^{2}$, M. Nasr ${ }^{1}$, N. M. Abd El Hamid ${ }^{3}$, H. Kamel ${ }^{2}$, A. Abd El Samie El Sherif ${ }^{2} .{ }^{1}$ Faculty of Medicine, Minia University, Rheumatology \& Rehabilitation, Minia, Egypt; ${ }^{2}$ Faculty of Medicine, Minia University, Department of Clinical and Chemical Pathology, Minia, Egypt; ${ }^{3}$ Minia Fever Hospital, Minia, Egypt

Background: Lupus nephritis (LN) is one of the most critical complications of systemic lupus erythematosus (SLE). Approximately $30-50 \%$ of SLE patients develop LN with 5 -year survival rate of about $70-80 \%$. Thus, finding reliable non-invasive biomarkers at the early stages of SLE is of great interest (1). Many studies focused on the association between microRNAs and the risk of LN. miRNA-146a (miR-146a) was one of the most promising circulating markers which was suggested recently for early diagnosis of SLE but its diagnostic relevancies regarding $\mathrm{LN}$ have not been extensively investigated.

Objectives: This study aims to test the expression of miR-146a in patients with LN in relation to Kallikrein-1 as another widely investigated diagnostic marker for $\mathrm{LN}$ along with other conventional measures.

Methods: One hundred and thirty subjects were enrolled in this study. They were divided into forty six patients with LN, forty four patients with SLE but without nephritis and forty healthy controls. The expression levels of miR-146a in peripheral blood mononuclear cells (PBMCs) were detected via RT-qPCR analysis. Besides, serum Kallikrein-1 levels were determined by ELISA. The diagnostic role of miR-146a and Kallikrein-1 in LN was evaluated by Receiver operating curve (ROC). The impact of miR-146a and Kallikrein-1 on renal disease was compared to albumin creatinine ratio, renal biopsy findings as well as renal SLEDAI.

Results: Levels of miR-146a were significantly lower in the plasma of $L N$ patients than both patients of SLE without LN and normal controls $(p<0.05)$. However, serum levels of Kallikrein-1 were significantly higher in LN patients when compared to SLE patients and normal population $(p<0.05)$. ROCs were conducted to assess the diagnostic values of both miR-146a and kallikrein-1. They revealed good diagnostic values with AUC of 0.888 and 0.913 respectively. Also, plasma miR-146a was observed to be negatively associated with serum creatinine, proteinuria as well as SLEDAI score $(p<0.01)$ while serum Kallikrein-1 was positively correlated with them $(p<0.05)$ and inversely correlated with miR-146a $(p$ $<0.01$ ).

Conclusion: The expression levels of miR-146a are reduced in SLE patients with more reduction with $L N$. Therefore, miR-146a could be considered as potential biomarker for detecting LN either alone or in combination with Kallikrein-1. However, more studies are required.

References:

[1] Soliman S. And Mohan C. Lupus nephritis Biomarkers.Clin Immunol.2017 Dec; 185: 10-20.

Disclosure of Interests: None declared

DOI: 10.1136/annrheumdis-2020-eular.5510

\section{\begin{tabular}{|l|l}
\hline FRI0186 JOINT INVOLVEMENT AND DISEASE ACTIVITY IN \\
\hline
\end{tabular} SYSTEMIC LUPUS ERYTHEMATOSUS PATIENTS: CALCULATION OF SWOLLEN TO TENDER JOINT COUNT RATIO IN A REAL WORLD COHORT IN THE US}

C. Cavanaugh ${ }^{1}$, G. Donadio ${ }^{1}$, K. Starzyk ${ }^{1}$, M. Behling ${ }^{1}$, G. Curhan ${ }^{1}$, R. Gliklich ${ }^{1}$. ${ }^{1} \mathrm{OM} 1$, Inc., Boston, United States of America

Background: Joint swelling and tenderness are common in patients with systemic lupus erythematosus (SLE). Swollen to tender joint count ratio (STR) is an index originally used in rheumatoid arthritis (RA) which assesses severity of disease activity based on 28 joint counts [1]. In RA, STR is a predictor of treatment response with a higher score indicating greater likelihood of responding.

Objectives: To characterize SLE patients in a real-world cohort based on disease activity as defined by STR.

Methods: The OM1 SLE Registry (OM1, Boston, MA) follows more than 37,000 SLE patients longitudinally with deep clinical data, including laboratory, patient-reported and disease activity information, and linked administrative claims, starting from 2013. Patients $\geq 16$ years of age with swollen and tender joint counts based on 28 joints on the same encounter were included. STRs were calculated by inserting 1 if the denominator was 0 [2]. Patients were categorized by first available STR as having low (STR $<0.5)$, moderate $(0.5 \leq \mathrm{STR} \leq 1.0)$, and high $(S T R>1.0)$ disease activity. Clinical characteristics were summarized by disease activity group. Definitions of SLE treatments were based on 2019 EULAR recommendations [3].

Results: As of December 2019, there were 9,919 patients with at least one STR available in the OM1 SLE Registry. STR was low in $80.4 \%$, moderate in $12.2 \%$, and high in $7.4 \%$ of patients. Mean age overall was 52.1 years (standard deviation: 14.8 ), $92.1 \%$ were female, and $71.8 \%$ of 7,730 patients with known race were white. Clinical characteristics by STR group are described in Table 1. Antimalarial use decreased and immunosuppressant use increased with increasing STR. Use of select disease-modifying antirheumatic drugs 
(DMARDs) was higher among patients with moderate or high STR. Lupus nephritis was more common in patients with low STR. A higher proportion of patients with moderate STR had osteoarthritis. The proportion of patients with anxiety and depression decreased with increasing STR. On average, patient and physician global assessments from MDHAQ were higher for patients with moderate STR.

Table 1. Clinical characteristics of patients with SLE by swollen:tender joint count ratio group

\begin{tabular}{lccc}
\hline & $\begin{array}{c}\text { Low } \\
\text { STR }<0.5 \\
(\mathrm{~N}=7,970)\end{array}$ & $\begin{array}{c}\text { Moderate } \\
\mathbf{0 . 5} \leq \mathrm{STR} \leq 1.0 \\
(\mathrm{~N}=1,211)\end{array}$ & $\begin{array}{c}\text { High } \\
\text { STR }>1.0 \\
(\mathrm{~N}=738)\end{array}$ \\
\hline Treatment prior to STR, $\mathrm{n}(\%)$ & & & \\
$\quad$ Antimalarial & $5,106(64.1 \%)$ & $702(58.0 \%)$ & $427(57.9 \%)$ \\
$\quad$ Biologics (belimumab or rituximab) & $662(8.3 \%)$ & $113(9.3 \%)$ & $70(9.5 \%)$ \\
Immunosuppressants & $2,310(29.0 \%)$ & $398(32.9 \%)$ & $252(34.1 \%)$ \\
Select DMARDs & $635(8.0 \%)$ & $165(13.6 \%)$ & $94(12.7 \%)$ \\
$\quad$ Steroids & $4,437(55.7 \%)$ & $785(64.8 \%)$ & $434(58.8 \%)$ \\
Disease conditions prior to STR, $\mathrm{n}(\%)$ & & & \\
Anxiety & $266(3.3 \%)$ & $25(2.1 \%)$ & $12(1.6 \%)$ \\
Depression & $1,127(14.1 \%)$ & $149(12.3 \%)$ & $80(10.8 \%)$ \\
Lupus nephritis & $984(12.3 \%)$ & $117(9.7 \%)$ & $72(9.8 \%)$ \\
Osteoarthritis & $2,336(29.3 \%)$ & $393(32.5 \%)$ & $193(26.2 \%)$ \\
Osteoporosis & $631(7.9 \%)$ & $95(7.8 \%)$ & $47(6.4 \%)$ \\
MDHAQ, N & 1,991 & 388 & 230 \\
MDHAQ, mean (SD) & & & \\
Patient global assessment (0-10) & $4.5(2.9)$ & $5.3(2.7)$ & $4.4(2.8)$ \\
Physician global assessment (0-10) & $2.8(2.7)$ & $3.8(2.6)$ & $2.8(2.3)$ \\
\hline
\end{tabular}

Conclusion: Differences in treatments received were apparent between patients of varying disease activity groups with trends towards increased use among patients with higher disease activity. Additional research is needed to determine the utility of this measure for assessing SLE-related outcomes.

References:

[1] Cipriano et al., Reumatismo 2015 Sept 16;67(2):62-7

[2] Hammer HB et al., Arthritis Rheumatol 2016; 68 (suppl 10)

[3] Fanouriakis et al., Ann Rheum Dis. 2019;78:736-745

Disclosure of Interests: None declared

DOI: 10.1136/annrheumdis-2020-eular.4514

\section{FRI0187 IMMUNOGENICITY OF 23-VALENT POLYSACCHARIDE PNEUMOCOCCAL VACCINE IN PATIENTS WITH SYSTEMIC LUPUS ERYTHEMATOSUS: EFFECT OF BIOLOGIC THERAPY ON THE VACCINAL RESPONSE}

G. Tarasova ${ }^{1}$, B. Belov ${ }^{1}$, M. Cherkasova ${ }^{1}$, S. Solovyev ${ }^{1}$, E. Aseeva ${ }^{1}$, T. Reshetnyak', T. Popkova ${ }^{1}{ }^{1}$ V.A. Nasonova Research Institute of Rheumatology, Moscow, Russian Federation

Background: Vaccination with 23-valent polysaccharide pneumococcal vaccine (PPV-23) in systemic lupus erythematosus (SLE) provides the prevention of severe respiratory infections in patients receiving immunosuppressive therapy. The importance of this vaccination significantly increases before and during treatment with biologics.

Objectives: The aim of the study was to evaluate the immunogenicity of PPV-23 in SLE patients.

Methods: The study included 52 patients with SLE, including 44 women and 8 men, aged 19 to 68 years. The duration of the disease varied from 9 months to 39 years. At the time of vaccination 7 patients had high, $10-$ moderate, 30 - low activity of the disease according to SLEDAI 2K, and 5 had remission. 50 patients received glucocorticoids (GC) $5-30 \mathrm{mg} /$ day equivalent to prednisone, 39 - hydroxychloroquine (GCH), 29 - cytostatics (CS), 20 - biologics: 10 - rituximab (RTM), 10 - belimumab (BLM). 1 dose $(0.5 \mathrm{ml})$ of PPV23 was administered subcutaneously. During the visits, standard clinical and laboratory tests were performed, and the level of antibodies $(\mathrm{Ab})$ to S.pneumoniae in blood serum was determined (VaccZymeTMPCPIg 2 kits - The Binding Site Ltd, Birmingham, UK).

Results: In 1-2 months after the vaccination $78.7 \%$ of patients had a significant (more than 2 times compared to baseline) increase in the concentration of $A b$ to the pneumococcal cell wall polysaccharides. A year after vaccination, $61.5 \%$ of patients ("responders") had a significant increase in the concentration of anti-pneumococcal Ab. 20 (38.5\%) of 52 patients were considered "non-responders". Median concentration of anti-pneumococcal Ab was 67[42.6; 105.8] mg/l at visit 1 (initially), 405[143.5; 468.4] mg/l at visit 2 (in 1-2 months), 166.9[77.5; $377.4] \mathrm{mg} / \mathrm{l}$ at visit 3 (in 12 months).

There were clear differences in the degree of the vaccinal response depending on the therapy: in 20 patients receiving biologics full vaccinal response was achieved significantly less frequently than in patients who did not receive these drugs ( $40 \%$ and $75 \%$, respectively), $\mathrm{p}=0.02$. There were no obvious differences in the vaccinal response during treatment with RTM and BLM $(40 \%$ of responders in both groups). The vaccinal response significantly decreased during treatment with biologics in combination with $\mathrm{GC}+/-\mathrm{GCH}$ ( $50 \%$ of responders). The lowest vaccinal response was observed in patients receiving biologics in combination with $\mathrm{GC}$ and $\mathrm{CS}+1$ - GCH (33.3\% of responders).

The analysis of the degree of the vaccinal response depending on the timing of vaccination and the time of biologics infusion was carried out. In the first group $(n=6)$, vaccination was carried out at the optimal time in accordance with the recommendations of EULAR (2020). In the second group $(n=14)$ vaccination was carried out in suboptimal time: during regular treatment with BLM $(n=6), 1$ week before the next introduction of RTM $(n=2), 3-5$ months after the last introduction of RTM (5), 1 week before the next introduction of RTM (n=1), 20 days after the BLM termination $(n=1)$. In the first group with optimal vaccination terms, the number of responders was $66.7 \%$, in the second group with suboptimal terms $-28.6 \%, p=0.27$

Conclusion: Sufficient immunogenicity of PPV-23 was shown in SLE patients receiving immunosuppressive therapy. The negative impact of biologics on the vaccinal response was confirmed, especially if the vaccination was not performed at the optimal time in relation to the infusion of the drug or during monthly administration of BLM. If optimal vaccination terms are maintained during the treatment with or initiation of biologics ( 6 months after the last administration of RTM and 1 month before the next (or first) administration of RTM, 4 months after the last and 1 month before the next (or first) administration of BLM), the number of responders increases significantly. The lowest vaccinal response was obtained in patients receiving combined immunosuppressive therapy with biologics + GC+CS.

Disclosure of Interests: None declared

DOI: 10.1136/annrheumdis-2020-eular.2596

\begin{tabular}{|l|l|}
\hline FRI0188 & THE CLINICOPATHOLOGICAL SIGNIFICANCE \\
OF MODIFIED NATIONAL INSTITUTES OF \\
HEALTH ACTIVITY AND CHRONICITY SCORING \\
SYSTEM IN LUPUS NEPHRITIS; A MULTICENTER \\
RETROSPECTIVE STUDY
\end{tabular}

T. Zoshima ${ }^{1}$, S. Hara ${ }^{1}$, M. Kawano ${ }^{1}{ }^{1}$ Kanazawa University, Rheumatology, Kanazawa, Japan

Background: The revised International Society of Nephrology/Renal Pathology Society (ISN/RPS) Classification of lupus nephritis (LN) 2018 defined a modified National Institutes of Health activity and chronicity scoring system for all LN classes [1]. As this was not arrived at by an evidence-based approach, its clinicopathological significance including prognostic value should be validated [1]. Furthermore, though the activity index included wire-loop lesion and hyaline deposits (WL), we previously demonstrated that WL was associated with serological immune abnormality, but not renal prognosis [2].

Objectives: We conducted this study to clarify the relationships of modified activity score (AS) and chronicity score (CS) to clinical parameters at the time of renal biopsy and renal and life prognoses, and also to investigate the impact of AS without WL.

Methods: We enrolled 138 Japanese LN patients subjected to renal biopsy in 11 hospitals from 2000 to 2019 . We measured clinical findings at the time of renal biopsy, and determined the presence of comorbidities. We also measured serum creatinine and estimated glomerular filtration rate (eGFR) at the last patient visit, and recorded medications prescribed for LN. Renal biopsy findings were classified by the modified ISN/RPS classification 2018 including AS and CS for all LN classes. On stepwise multivariate analysis, we applied the variables with significant differences in univariate comparisons. The primary endpoint was chronic kidney disease (CKD; eGFR $<60 \mathrm{ml}$ $\mathrm{min} / 1.73 \mathrm{~m}^{2}$ ) and/or death.

Results: Of 138 patients (116 females; median 39 years old), class I, II, III, IV, and $\mathrm{V}$ included 2 (1.4\%), 13 (9.4\%), 43 (31.2\%), 69 (50.0\%), and 11 (8.0\%), respectively. Median AS, AS without WL (AS-WL), and CS were 4, 3, and 2 , respectively. $A S \geq 5$ group ( 61 patients, $44.2 \%$ ) had higher proteinuria, hematuria and serum anti-ds DNA antibodies levels and lower serum total protein (TP) and C3 levels than AS $<5$ group. CS $\geq 3$ group ( 58 patients, $42 \%$ ) had higher age, proteinuria, serum $\mathrm{C} 3$ levels, and frequency of hypertension (HT) and lower eGFR and serum anti-ds DNA antibodies and IgG levels than $\mathrm{CS}<3$ group. Multiple regression analysis revealed significant associations between AS and hematuria, TP and C3 ( $\beta=0.312,-0.281,-0.213 ; p<0.001,0.001,0.009)$, and between CS and age $(\beta=0.300 ; p=0.010)$. Next, patients who achieved the primary endpoint had higher age, frequencies of HT and hyperlipidemia and lower eGFR serum TP and IgG levels than patients who did not. Observation period (median 36 vs 47 months, $p=0.696$ ) and medications for $L N$ did not differ between these groups. Cox regression analysis revealed significant associations of prognosis 